\title{
Sarcoidosis and cutaneous lymphoma: What is the relationship? ${ }^{*}$
}

\author{
Vanessa Gargallo ${ }^{1}$ \\ José Luis Rodríguez-Peralto ${ }^{3}$
}

\author{
Jéssica González Ramos² \\ Concepción Postigo ${ }^{1}$
}

\section{Dear Editor,}

A 30-year-old Caucasian woman with history of chronic active systemic sarcoidosis for 14 years, presented at our dermatology department complaining of recurrent ulcerative papules and nodules that had begun four years ago. Sarcoidosis, involving the lungs, lymph nodes, kidney (interstitial nephritis), skin (panniculitis) and eyes (uveitis), was diagnosed by ganglionar and renal biopsy and treated with oral steroid and methotrexate. The papules, located mainly on palms but also on legs and gluteal area, became ulcerated and tend to self-resolve within some weeks, leaving a scar whilst new self-resolving lesions appeared (Figure 1). Biopsy showed a diffuse atypical lymphocytic proliferation involving the papillary dermis, the reticular dermis and the subcutaneous tissue. Immunohistochemistry was CD3+, CD43+ and CD4+ with groups of positive CD30 cells (Figure 2). An extended clinical and radio-
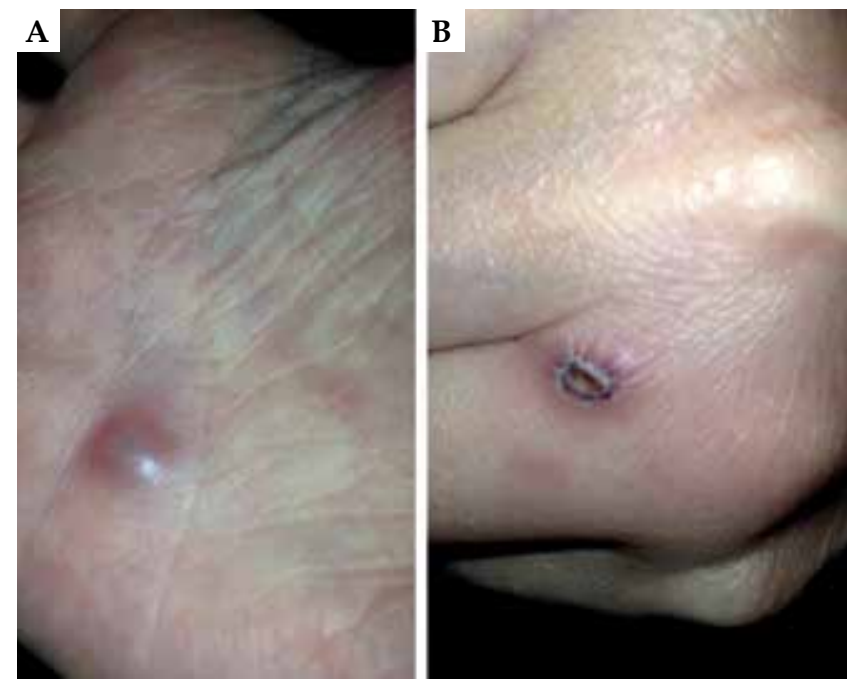

Figure 1: Clinical picture of the self-resolving lesions. A. Papulo-nodular lesion located on the palm of the right hand. B. Papulo-nodular lesion, ulcerated at its center, located on right hand logical study was performed, dismissing the possibility of systemic involvement. Biopsy findings and clinical history were compatible with lymphomatoid papulosis (LyP) type A and she was treated with low dose methotrexate and steroid cream. One year later the lesions became persistent and did not disappear spontaneously (Figure 3). Biopsy was equivalent to the previous. Five months later an infiltrated mass of $7 \mathrm{~cm}$ on her left groin appeared associated to groin, iliac and retroperitoneal lymphadenopathies. Biopsy of the mass and lymphadenopathies showed a tumoral infiltration of large atypical intensely eosinophilic lymphocytes with irregular nuclei accompanied by an inflammatory infiltrate. The tumoral cells where $\mathrm{CD} 3+, \mathrm{CD} 4+, \mathrm{CD} 30+, \mathrm{CD} 45+$ focally, ALK-, and had a high proliferative index (Ki.67 80\%). Hence, the diagnosis changed to ALK- primary cutaneous anaplastic large cell lymphoma with lymph node involvement. Chemotherapy with cyclophosphamide, doxorubicin, etoposide, vincristine and prednisone (CHOEP) was initiated but it was suspended on the 5 th course due to progression. Then, on second line, the patient received the AIEOP LNH-97 protocol, without response, initiating brentuximab with partial remission and performing later a bone marrow transplantation. The relationship between sarcoidosis and lymphoma seems not to be casual and the coexistence of both conditions is not infrequent. ${ }^{1}$ In the 1960's Bichel and Brincker suggested a possible association of lymphoma and sarcoidosis, using the term sarcoidosis-lymphoma syndrome. ${ }^{2}$ Bonifazi et al conducted a meta-analysis concluding that there is an increased risk of hematological malignancies and a higher overall risk of lymphoma in patients with systemic chronic active sarcoidosis. ${ }^{1}$ In spite of these findings, the relationship between sarcoidosis and cutaneous lymphoma including mycosis fungoides (MF) has not been studied yet. Several case reports describing concurrence of MF and sarcoidosis disease have been issued. ${ }^{3,4}$ This diagnosis is complicated because sarcoidal tissue reactions associated with cutaneous $\mathrm{T}$ cell lymphomas can occur, and some types of MF or anaplastic large cell CD30 lymphoma can resemble sarcoid granulomas clinically and histologically. ${ }^{2,5}$ Gelfand et $\mathrm{al}^{5}$ reported a case similar to ours: a patient with systemic sarcoidosis that presented at diagnosis with

Received on 07.07.2016

Approved by the Advisory Board and accepted for publication on 15.07.2016

Study conducted at Hospital Universitario 12 de Octubre and Hospital Universitario La Paz- Madrid, Spain.

Financial Support: None.

Conflict of Interest: None.

Dermatology Department. Hospital Universitario 12 de Octubre - Madrid, Spain.

Dermatology Department. Hospital Universitario La Paz - Madrid, Spain.

Pathology Department. Hospital Universitario 12 de Octubre - Madrid, Spain.

(C2017 by Anais Brasileiros de Dermatologia 


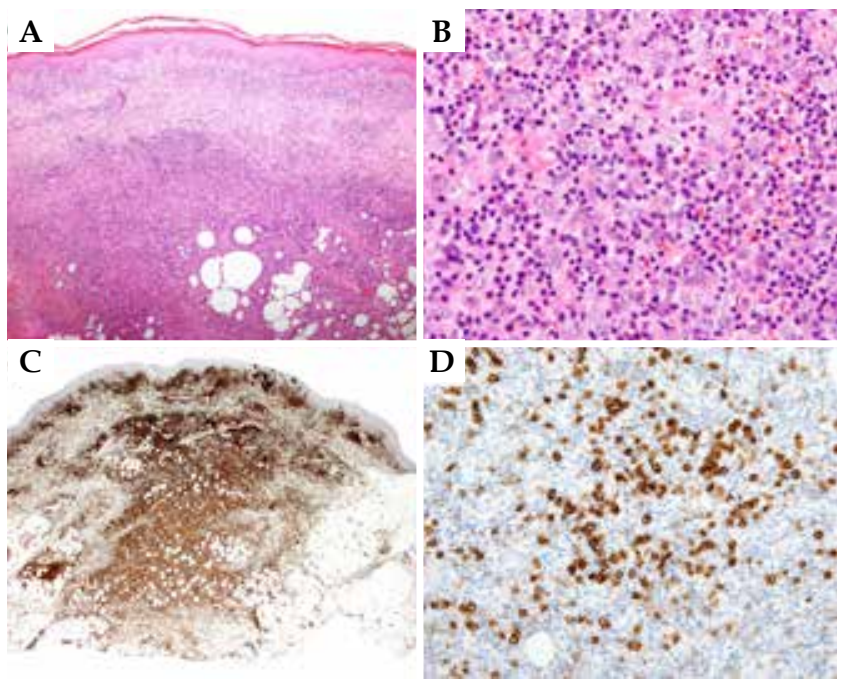

Figure 2: Histopathologic findings. A. View of dense infiltrate of lymphocytes in the papillary dermis, the reticular dermis and the subcutaneous tissue from biopsy taken from the hand. (Hematoxylin \& eosin $x 40)$. B. Detailed image of the infiltrate composed of small, medium and large atypical lymphocytes. (Hematoxylin \& eosin x100). C. Immunohistochemical findings with CD4+ T cells (Hematoxylin \& eosin $x 20)$. D. Immunohistochemical findings with groups of CD30+ cells (Hematoxylin \& eosin x100)

self-resolving papulo-nodular necrotic lesions. Biopsy demonstrated the presence of anaplastic cells with epidermotropism, CD30+, $\mathrm{CD} 3+, \mathrm{CD} 43+$, and CD8+. The initial lesions mimicked a LyP type $\mathrm{D}$, but afterwards they became persistent and progressive. Intriguingly, as in our patient, many of these lesions were located on the palms, which is an unusual location for LyP. On follow-up, the patient developed pulmonary involvement and died of progressive epidermotropic CD8+/CD4- primary cutaneous CD30+ lymphoproliferative disorder. Several theories have been set forth to explain the higher incidence of lymphoma when chronic active sarcoidosis is present. Organs targeted by inflammatory conditions can have an increased cancer risk. ${ }^{1}$ The lymphocytes in the involved tissues in sarcoidosis' immune inflammatory response have an increased mitotic activity, which can increase the possibility of mutation and
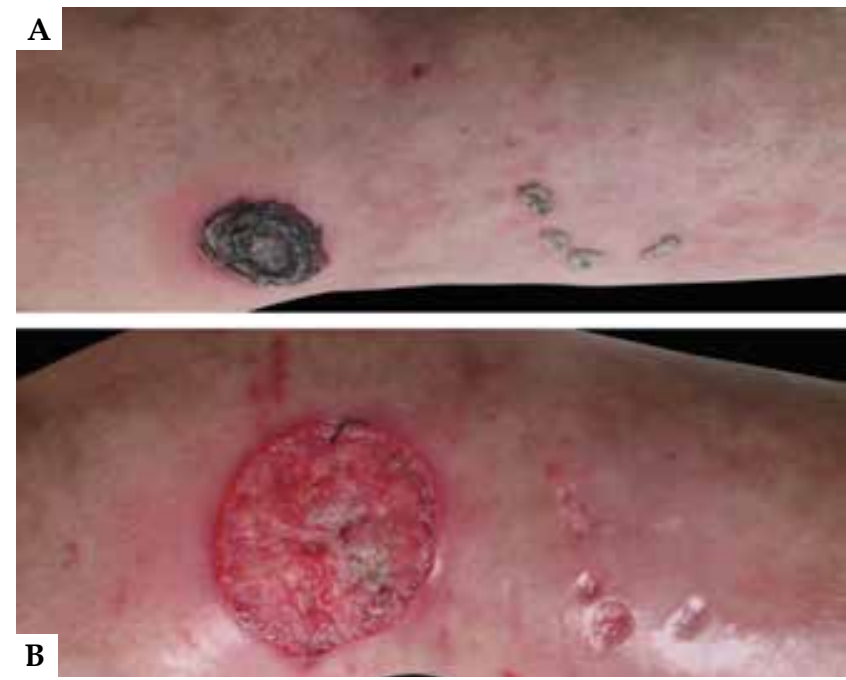

Figure 3: Clinical picture of the persistent lesions. A. Large ulcerative plaque of $3 \mathrm{~cm}$ on the leg accompanied by other smaller papulo-necrotic lesions. B. Observe the growing and persistence of the same lesions.

malignant transformation. ${ }^{2,4}$ Exposure to immunomodulatory treatments seems to contribute to malignant lymphoproliferation too. ${ }^{1}$ Finally, sarcoidosis is accompanied of immunological changes that lead to a dysfunctional T-cell activity such as a decrease of CD8+ cells or an activation of CD4+ cells, anomalous cytokine production (IL-6, TNFalfa, TGFbeta), reduced cellular immunity (anergy) that can increase lymphoma risk. ${ }^{1,2}$ Sarcoidosis is a T-cell type 1 (Th1) disease, and CD30+ lymphomas are a Th2 disease. Due to the antagonistic nature of the immunologic basis of both diseases, their coexistence can be a therapeutic challenge since treatments such as interferon-alfa can improve T-cell lymphoma whereas aggravate sarcoidosis. ${ }^{5}$ Although there is a relationship between sarcoidosis and lymphoma, it is still unclear if it exists with cutaneous lymphoma. However, is important to remind that chronic active sarcoidosis and LyP increase lymphoma risk and the concurrence of both should alert of the possibility of developing an aggressive cutaneous lymphoma. $\square$

\section{REFERENCES}

1. Bonifazi M, Bravi F, Gasparini S, La Vecchia C, Gabrielli A, Wells AU, et al. Sarcoidosis and cancer risk: systematic review and meta-analysis of observational studies. Chest. 2015;147:778-91.

2. Suvajdzic N, Milenkovic B, Perunicic M, Stojsic J, Jankovic S. Two cases of sarcoidosis-lymphoma syndrome. Med Oncol. 2007;24:469-71.

3. McFarland JP, Kauh YC, Luscombe HA. Sarcoidosis associated with mycosis fungoides. Arch Dermatol. 1978;114:912-5.

4. Schmuth M, Prior C, Illersperger B, Topar G, Fritsch P, Sepp N. Systemic sarcoidosis and cutaneous lymphoma: is the association fortuitus? $\mathrm{Br} J$ Dermatol. 1999;140:952-5.

5. Gelfand JM, Wasik MA, Vittorio C, Rook A, Junkins-Hopkins JM. Progressive epidermotropic CD8+/CD42 primary cutaneous CD30+ Iymphoproliferative disorder in a patient with sarcoidosis. J Am Acad Dermatol. 2004;51:304-8.

\author{
MAILING ADDRESS: \\ Vanessa Gargallo \\ Avenida de Córdoba s $/ n$ \\ 280041 Madrid (12 de Octubre University Hospital) \\ Paseo de la Castellana, 261 \\ 28046 Madrid (La Paz University Hospital) \\ E-mail:vgmoneva@gmail.com
}

How to cite this article: Gargallo-V, González Ramos J, Rodríguez-Peralto JL, Postigo C. Sarcoidosis and cutaneous lymphoma: What is the relationship? An Bras Dermatol. 2017;92(1):158-9. 